\title{
Classification of Voltage Sag Causes using Probabilistic Neural Network and Hilbert - Huang Transform
}

\author{
M. Manjula \\ Member, IEEE \\ Department of Electrical Engineering \\ Osmania University, Hyderabad
}

\author{
A.V.R.S. Sarma \\ Member, IEEE \\ Department of Electrical Engineering \\ Osmania University, Hyderabad
}

\begin{abstract}
Any power quality disturbance waveform can be seen as superimposition of various oscillating modes. It becomes necessary to separate different components of single frequency or narrow band of frequencies from a non stationary signal to identify the causes which contribute to power quality disturbances. In this paper a method is proposed to detect and classify voltage sag causes based on Empirical Mode Decomposition (EMD) with Hilbert Transform ( called HilbertHuang Transform) and Probabilistic Neural Network (PNN). The key feature of EMD is to decompose a non stationary signal into mono component signals called Intrinsic Mode Functions (IMFs). Further the Hilbert transform of each IMF provides frequency information evolving with time and variation in magnitude and phase due to oscillation at different time scales and locations. The characteristic features of the first three IMFs of each disturbance waveform are obtained. Finally PNN is used to classify the characteristic features for identification of voltage sag causes. Three voltage sag causes are taken for classification (i) Three phase short circuit (ii) Starting of induction motor and (iii) Three phase transformer energization. Results show that the classifier can detect and classify the voltage sag causes efficiently.
\end{abstract}

\section{Keywords}

Empirical mode decomposition, intrinsic mode functions, hilbert transform, probabilistic neural network, voltage sag causes.

\section{INTRODUCTION}

Power quality has become an important issue of electrical power system operation in recent years [1], because of the increased use of modern power electronic devices that are very sensitive to voltage disturbances. The most commonly attributed power quality disturbances are voltage sag, voltage swell, interruptions, voltage flicker, voltage fluctuations and harmonics. To mitigate The power quality disturbances, one has to know about the sources of power system disturbances or the actual causes behind them. This can be achieved by detecting and classifying the different power system disturbances. Disturbance data and power quality data have become important information both for statistical purposes and for decision making in mitigation devices. This requires the analysis of data and classification so that a lot of time could be saved if they are done automatically. Voltage sag is one of the most disturbing power quality problem. The voltage sags are mostly due to short circuit fault, starting of induction motor or due to transformer energizing. A simple way to analyze any signal is by Fourier Transform [2](FT). However it provides only frequency content, therefore this method is applicable for stationary signals. To over come this drawback, Short Time Fourier Transform (STFT) is proposed [3-4] which maps a signal into a two dimensional function of time and frequency. The STFT extracts time and frequency information, the disadvantage is that the size of the window is fixed for all frequencies. The wavelet analysis [5-7] represents a windowing technique with variable regions to overcome the deficiency. It provides a unified methodology to characterize power quality events by decomposing the signal into time and frequency resolution. A wavelet transform expands a signal in terms of trigonometric polynomial by using wavelets generated using transition (shift in time) and dilation (compression in time) of a fixed wavelet function. So wavelet function is localized both in time and frequency, yielding wavelet coefficients at different scales. The draw back of wavelet transform is that its ability to detect under noisy conditions is not accurate. The S-transform[8] on the other hand is an extension to wavelet transform and is based on moving and scalable localizing Gaussian window.

This paper presents the Empirical Mode Decomposition (EMD), introduced by Huang [9], together with Hilbert transform for extracting mono component and symmetric components using the varying instantaneous amplitude and frequency from multi component non stationary signals. These mono component signals are called the Intrinsic Mode Functions. The advantage of this method is that it does not require predetermined set of functions as in previous methods and it allows projection of a non stationary signal onto a time frequency plane using a mono component signals, from the original signal itself thus making it adaptive in nature.

This paper is organized into eight sections, section 2 gives introduction to EMD and its implementation on a distorted waveform to extract IMFs. Section 3 presents the application of Hilbert transform to the IMFs. Section 4 deals with three voltage sag causes and various features extracted from the respective IMFs. Section 5 explains the probabilistic neural network classification for voltage sag causes. Section 6 gives the results and discussion about the methodology. Section 7 gives the conclusions of the work. In the end section 8 gives the references. 


\section{EMPIRICAL MODE DECOMPOSITION}

When a signal is not stationary amplitude and frequency change with time as in the case of most power quality disturbances. Representing such a non-stationary signal as a combination of different sinusoidal signals will not be accurate and single frequency can not be defined. This accounts for a parameter which vary with time. So it is necessary to have a more flexible and extended notion of frequency. This gives rise to an idea of Instantaneous frequency. (IF) which means for a signal having a single frequency or narrow band of frequencies. Many of the power quality disturbances are non stationary and the concept of IF would be of great help. Therefore it becomes important for such an algorithm which separates different components of single frequency such that IF can be defined for each signal. Empirical mode decomposition is a method which extracts mono component and symmetric components from the non linear and non stationary signals by sifting process. The name, sifting, indicates the process of removing the lowest frequency information until only the highest frequency remains. The key feature of EMD is to decompose a signal into so called Intrinsic mode functions. These Intrinsic Mode Functions extracted from the original signal are mono component composing of single frequency or narrow band of frequencies. Huang et al. defined an oscillating wave as an IMF if it satisfies the following two conditions :

(a) For a data set, the number of extreme and the number of zero crossings must either be equal or differ at most by one.

(b) At any point, the mean value of the envelope defined by the local maxima and the local minima is zero.

The essential step of extracting an IMF is by iteratively conducting the sifting process as given below:

(i) The upper and the lower envelopes are constructed by connecting all the maxima and all the minima with cubic splines, respectively.

(ii) Take the mean of the two envelopes and let it be defined as $\mathrm{m}(\mathrm{t})$.

(iii) Subtract the mean $\mathrm{m}(\mathrm{t})$ from the original signal $\mathrm{x}(\mathrm{t})$ to get a component $h_{1}(t)$, where

$$
h_{1}(t)=x(t)-m(t)
$$

(iv) If $h_{1}(t)$ satisfies the two conditions of IMFs, then $h_{1}(t)$ is the first intrinsic mode function else it is treated as the original function and steps (i) - (iii) are repeated to get component $\mathrm{h}_{11}(\mathrm{t})$ such that

$$
h_{11}(t)=h_{1}(t)-m_{1}(t)
$$

(v) The above sifting process is repeated $\mathrm{k}$ times, $\mathrm{h}_{1 \mathrm{k}}(\mathrm{t})$ becomes an first IMF and be known as IMF1.

(vi) Separate IMF1 from $\mathrm{x}(\mathrm{t})$ and let it be $\mathrm{r}_{1}(\mathrm{t})$, such that

$$
r_{1}(t)=x(t)-h_{1 k}(t)
$$

(vii) Now taking the signal $\mathrm{r}_{1}(\mathrm{t})$ as the original signal and repeating the steps (i) - (vi) second IMF is obtained.

(viii)The above procedure is repeated $\mathrm{n}$ times and such $\mathrm{n}$ IMFs are obtained.

(ix) The stopping criterion for the decomposition process is when $r_{n}(t)$ becomes a monotonic function from which no more IMF can be extracted.

An example of voltage sag with harmonics is taken for implementation of EMD to extract intrinsic mode functions. The following figure 1 . shows the IMFs.
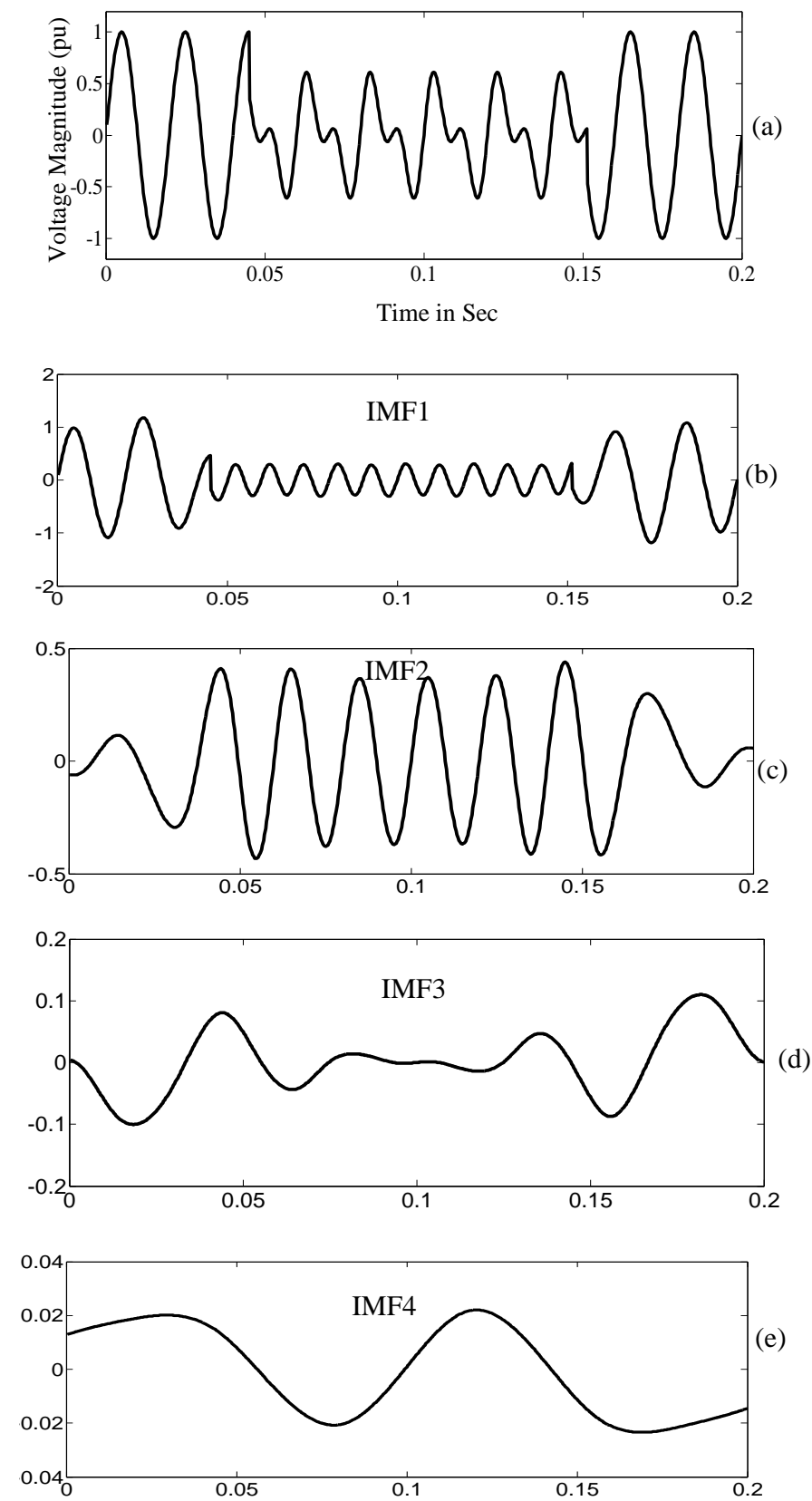


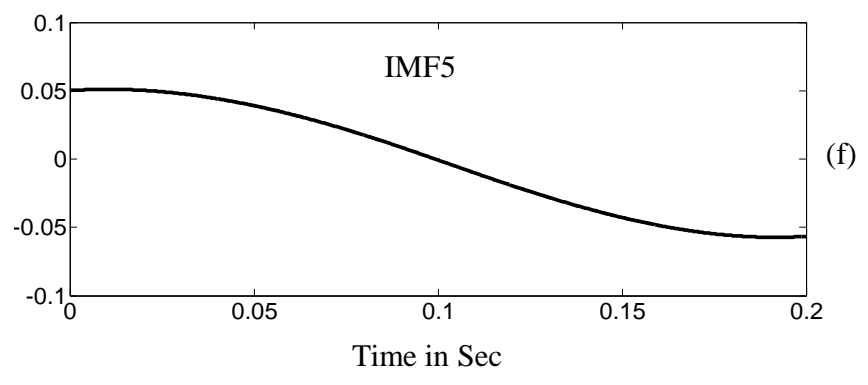

Figure 1. Empirical mode decomposition with corresponding IMFs (a) voltage sag with harmonics (b)-(f) IMFs.

\section{HILBERT TRANSFORM}

The Instantaneous frequency of each IMF is calculated by using the Hilbert Transform[10]. The Hilbert Transform of a real valued time domain signal $\mathrm{x}(\mathrm{t})$ is another real valued time domain signal, denoted by $\hat{x}(\mathrm{t})$, such that $\mathrm{z}(\mathrm{t})=\mathrm{x}(\mathrm{t})+\mathrm{j} \hat{x}(\mathrm{t})$ is an analytic signal. From $\mathrm{z}(\mathrm{t})$, one can define a magnitude function $\mathrm{A}(\mathrm{t})$ and a phase function $\theta(t)$, where the first describes the envelope of the original function $\mathrm{x}(\mathrm{t})$ versus time and $\theta(t)$ describes the instantaneous phase of $x(t)$ versus time.

The Hilbert transform of a real-valued function $x(t)$ extending over the range $-\infty<t<+\infty$ is a real-valued function $\hat{x}(\mathrm{t})$ defined by:

$$
\hat{x}(t)=H[x(t)]=\int_{-5}^{J} \frac{x(u)}{\pi(t-u)} d u
$$

Thus $\hat{x}(\mathrm{t})$ is the convolution integral of $x(t)$ and $(1 / \pi t)$, written as:

$$
\hat{x}(t)=x(t) *\left(\frac{1}{\pi t}\right)
$$

Like Fourier transforms, Hilbert transforms are linear operators. A useful point of view to understand and to compute the Hilbert Transform of $\hat{x}(\mathrm{t})$ is using the analytic signal $z(t)$ associated with $x(t)$, as

$$
z(t)=x(t)+j \hat{x}(t)
$$

that can be rewritten also as:

$$
z(t)=A(t) \times e^{\text {str }}
$$

where $A(t)$ is called the envelope signal of $x(t)$ and $\theta(t)$ is called the instantaneous phase signal of $x(t)$.

In terms of $x(t)$ and $\hat{x}(\mathrm{t})$, it is clear that:

$$
\begin{aligned}
& A(t)=\left[x^{3}(t)+j \hat{x}^{3}(t)\right]^{1 / \beta} \\
& \theta(t)=\tan ^{-1}\left[\frac{\hat{x}(t)}{x(t)}\right]
\end{aligned}
$$

and the "instantaneous frequency" is given by:

$$
f_{0}=\frac{1}{2 \pi} \tan ^{-1}\left[\frac{\hat{x}(t)}{x(t)}\right]
$$

\section{VOLTAGE SAG CAUSES AND THEIR FEATURE EXTRACTION}

\subsection{Fault - induced voltage sag (rectangular)}

Fault-induced voltage sags are considered as they are severe and cause problems to a large number of customers and propagate in the system. The magnitude of this type of voltage sag at a certain point in the system depends mainly on the type of the fault, the distance to the fault, the system configuration and the fault resistance. Its duration depends on the type of protection that is used. Faults are either symmetrical (three phase or three phase-to ground fault) or non-symmetrical (single phase or double phase or double phase-to-ground faults). Depending on the type of fault the magnitudes of the voltage sag of each phase might be equal (symmetrical fault) or unequal (non symmetrical faults). Here symmetrical fault is considered as it is balanced voltage sag. They are rectangular sags, voltage drops very fast and remains almost constant to a new lower value until protection operates. The characteristics of such sags are (1) Immediate recovery of voltage (2) The change in the phase angle and (3) No harmonic distortion. A three phase short circuit was applied on $11 \mathrm{Kv}$ power system network for different values of fault resistance (Rf) were simulated. The duration of fault applied and time of application were varied. The disturbance is referred as VSC1. All the power quality disturbances are generated using MATLAB/SIMULINK software. Figure 2.(a), shows waveform of the voltage sag due three phase short circuit (magnitude $(\mathrm{pu})),(\mathrm{b})$, voltage magnitude (rms), (c)-(h), corresponding IMFs.
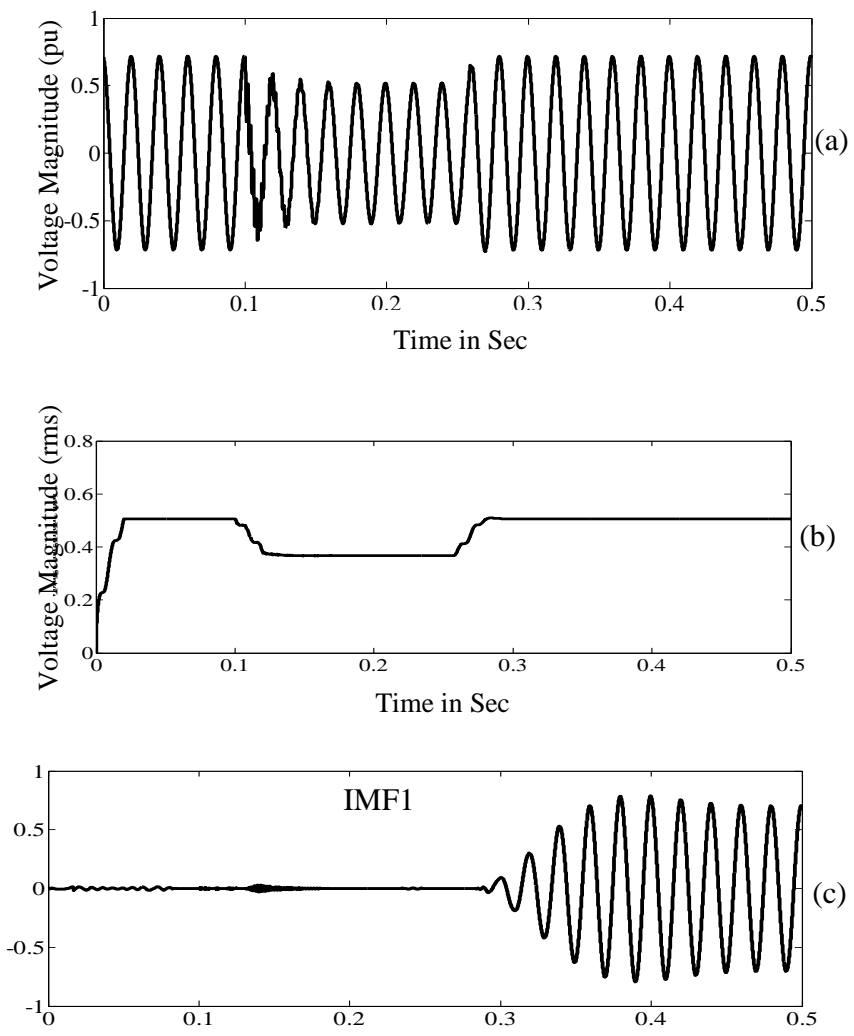

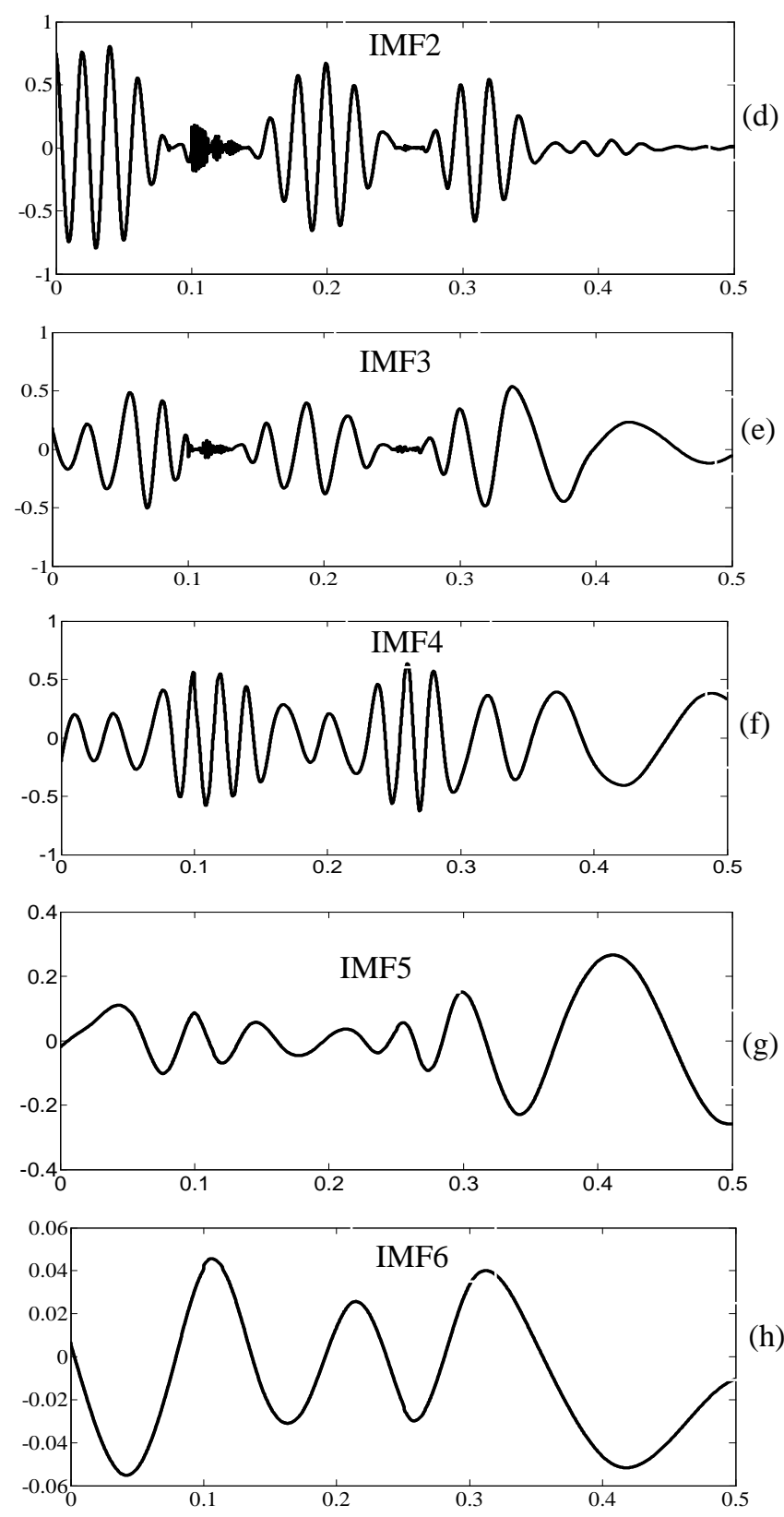

Figure 2. (a) voltage sag due to three phase short circuit $\left(R_{f}=10 \Omega\right),(b)$ voltage magnitude (rms), (c)-(h) IMFs .

\subsection{Voltage sag due to transformer energization (non-rectangular)}

Transformer saturation might occur during energizing or changes in the voltage at the transformer terminals. It causes nonrectangular voltage sags and temporary harmonic distortion. The voltage sag caused by transformer energization presents different RMS magnitude. The characteristics of such sags are (1) Unbalanced sag in all three phases (2) Shallow voltage sag (3)Gradual recovery of voltage (4) No phase angle shift (5) Harmonic distortions. In this case a $500 \mathrm{Kv}$ power system consisting of a $500 / 315 \mathrm{Kv}$ two winding transformer was energized for simulating the waveforms. The point on wave for energizing the transformer was varied. The simulations were carried out for star/delta, star/star, and delta/star connections. The disturbance is referred as VSC2. Figure 3. (a) Shows the voltage sag waveform due transformer energization (magnitude (pu)), (b), voltage magnitude (rms), (c)-(k) corresponding IMFs.
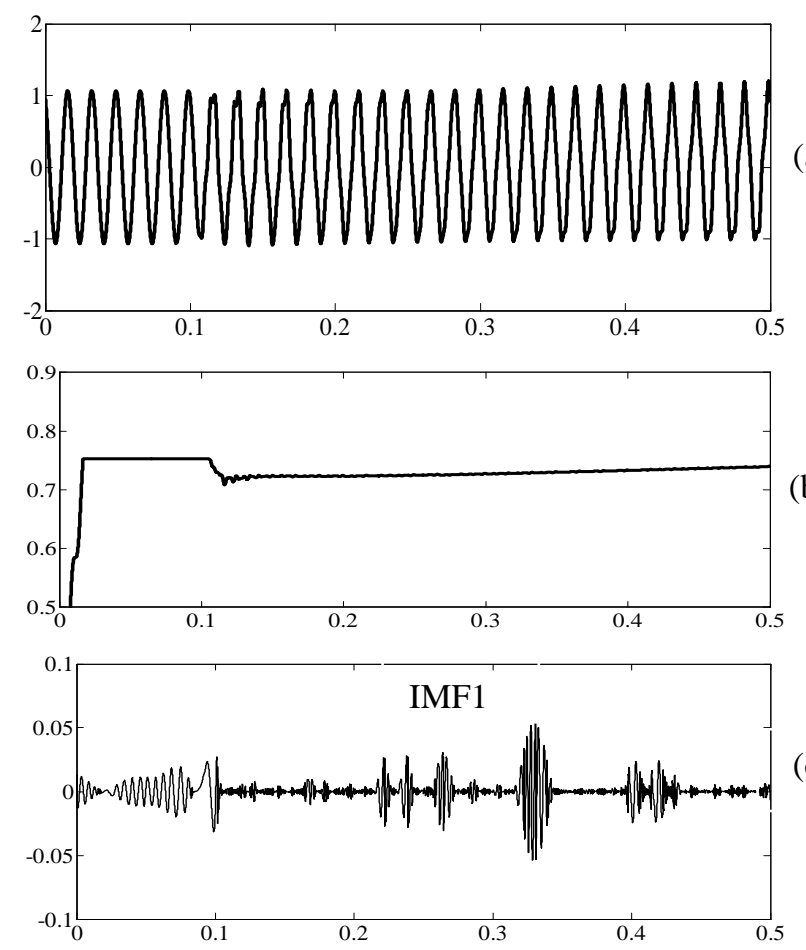

(c)
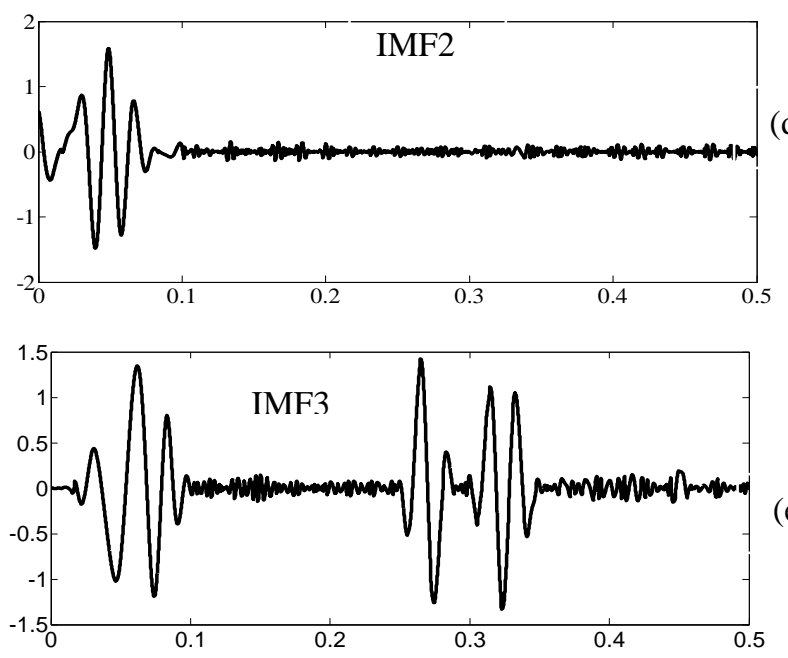

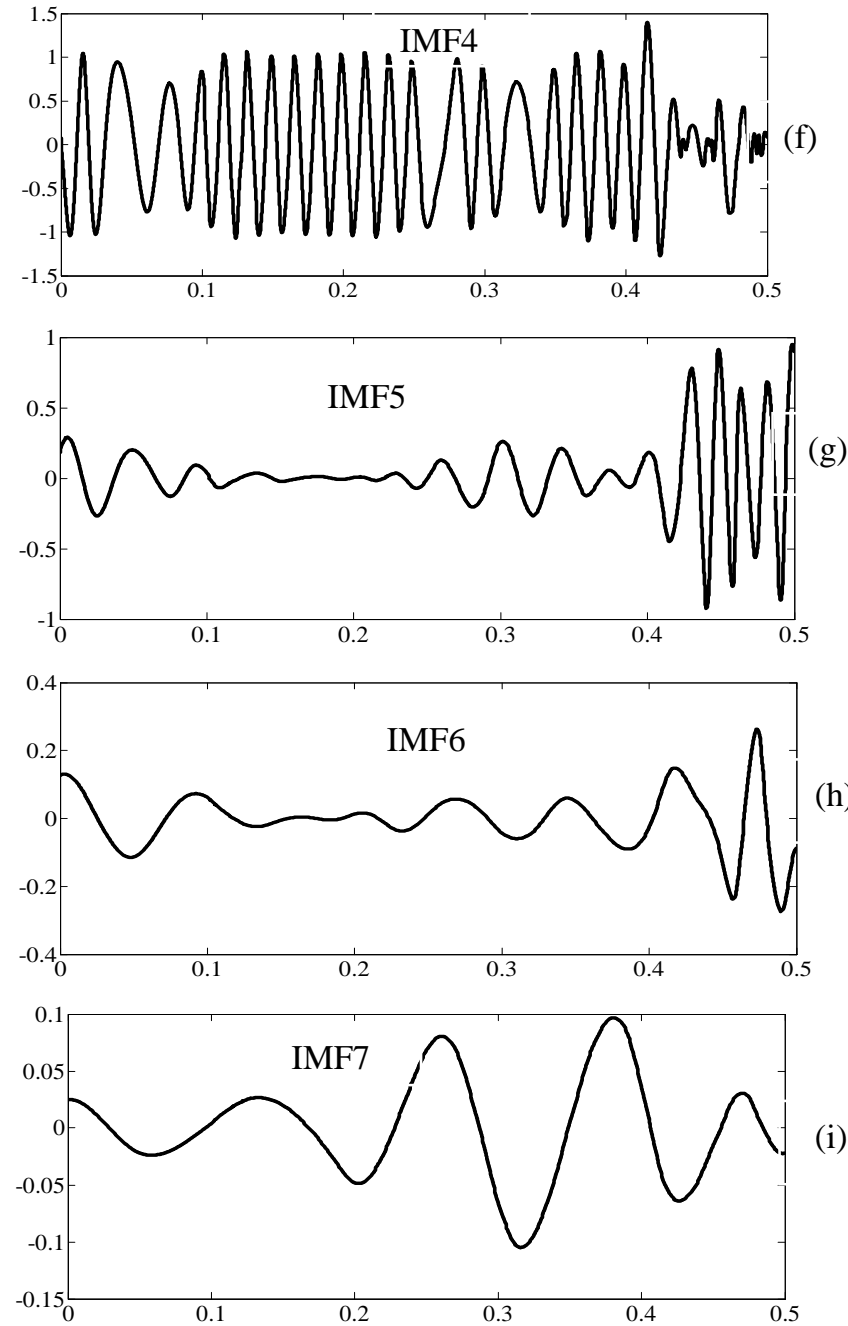

(i)
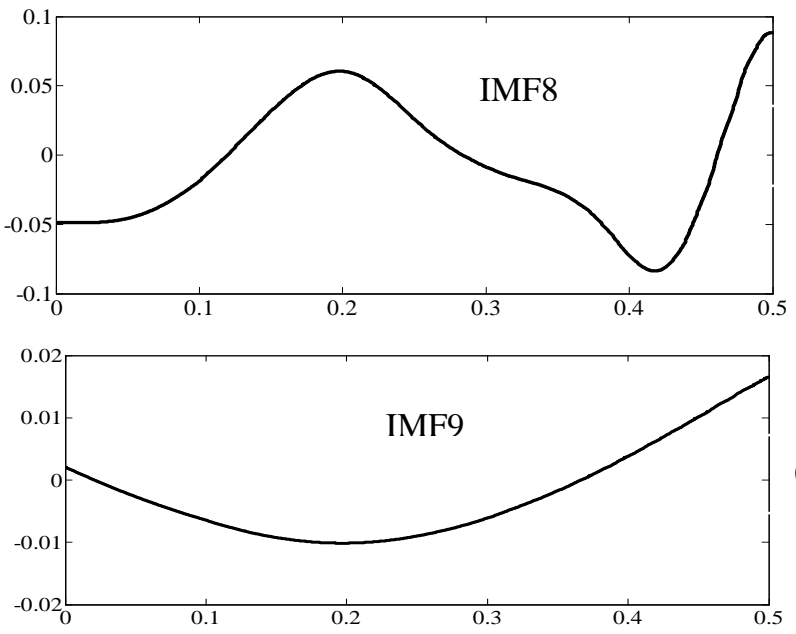

(j)
Figure 3. (a) voltage sag due to transformer energization (b) voltage magnitude (rms), (c)-(k) IMFs .

\subsection{Voltage Sag due to Induction Motor Starting(non-rectangular)}

During starting, motors draw approximately five-times their fullload running current, and at a very low power factor. This starting current causes shallow voltage sags. The magnitude of the voltage sag depends on the characteristics of the induction motor and the strength of the system at the point that the motor is connected. The characteristics of such sags are (1) Balanced sag in all three phases (2) Shallow voltage sag (3) Gradual recovery of voltage (4) No phase angle shift (5) No harmonic distortions. In this case a $400 \mathrm{v}$ power system network with the following 5.4, $10,20,50,100,150 \mathrm{HP}$ rating of the induction motor were considered. The disturbance is referred as VSC3. Figure 4.(a) shows the voltage sag waveform due starting of induction motor, (b) voltage magnitude (rms), (c)-(h) corresponding IMFs.

(h)

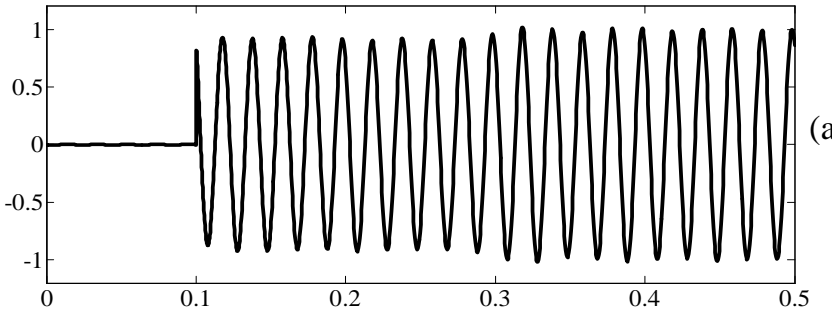

(a)
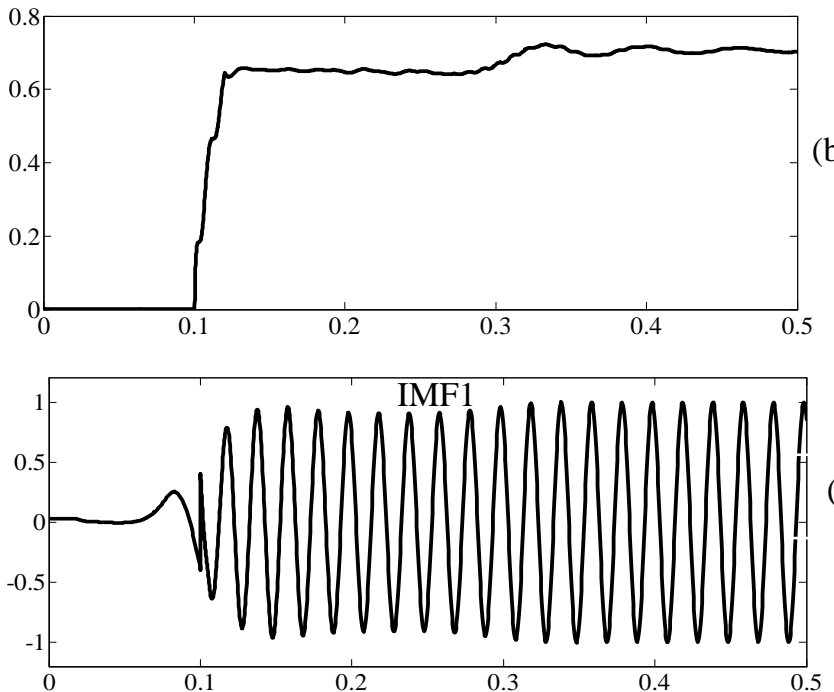

(c)

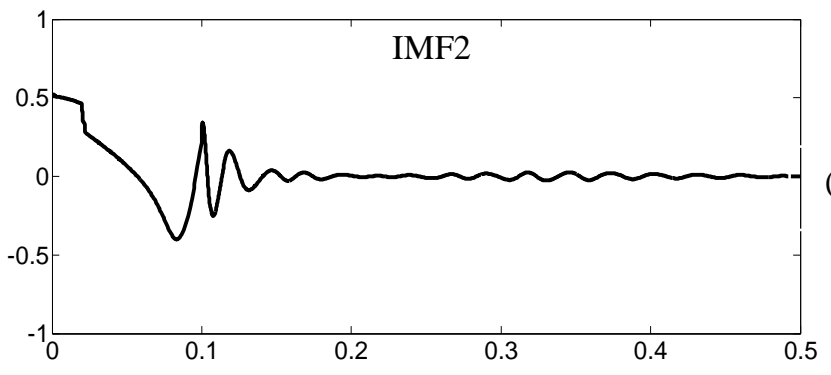




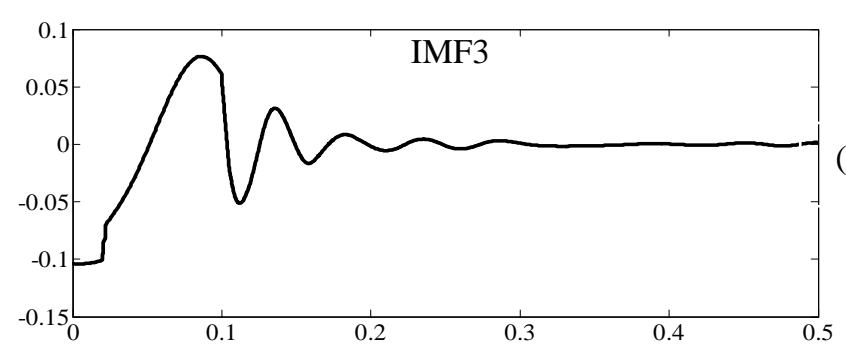

(e)
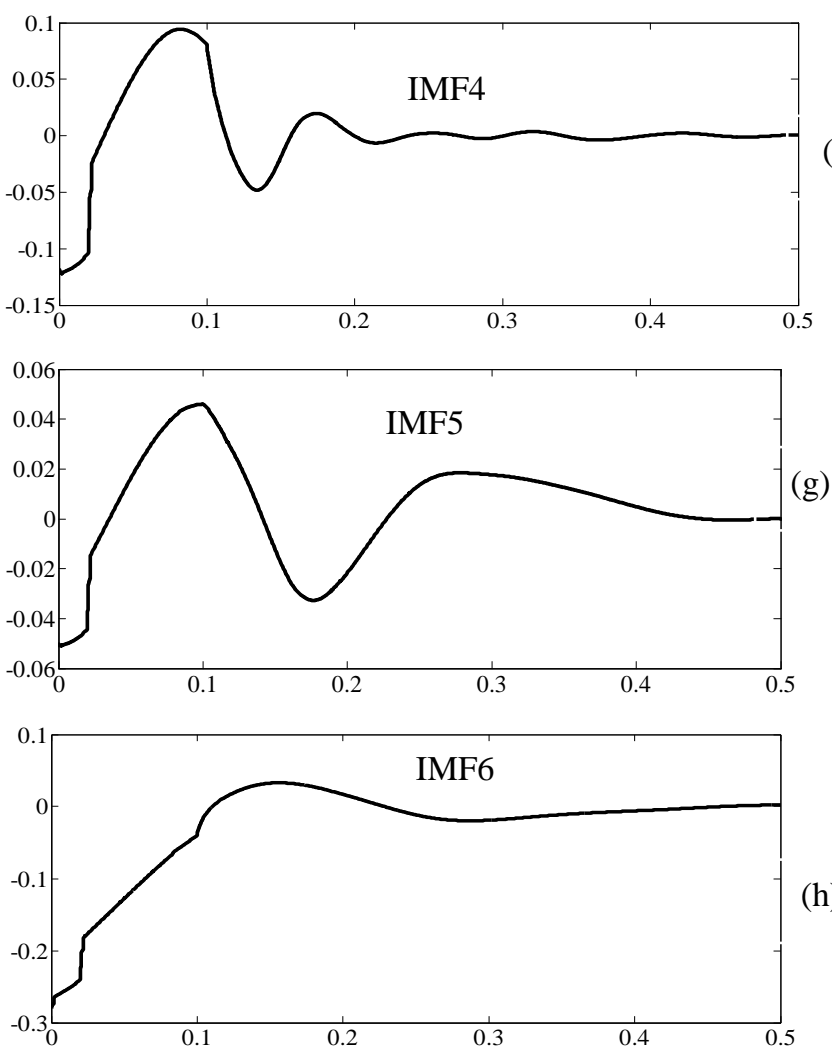

(h)

Figure 4. (a) voltage sag due to starting of induction motor (b) voltage magnitude (rms), (c)-(h) IMFs .

Feature exaction is a pre-processing operation that transforms a pattern from its original form to a new form suitable for further processing like classification of events. Features of the disturbance signal are extracted by applying the Hilbert transform to the IMFs obtained from the EMD. The first three IMFs are considered for the feature extraction, as the number of IMFs are not the same all the disturbances. The reason for this is, that the number of IMFs depends on the nature of the original signal. The following three features are

(a) Energy distribution

(b) Standard deviation of the amplitude

(c) Standard deviation of the phase.

Thus, we have nine features from the three IMFs for each disturbance.

\section{PROBABILISTIC NEURAL NETWORK FOR CLASSIFICATION OF VOLTAGE SAG CAUSES}

The probabilistic neural network [11] (PNN) is a supervised neural network that is widely used in the area of pattern recognition, nonlinear mapping, and estimation of the probability of class membership. It is closely related to Bayes classification rule, and Parzen nonparametric probability density function (PDF) estimation theory. The fact that PNNs offer a way to interpret the network's structure in terms of probability density functions is an important merit of this type of networks. The standard training procedure for PNNs requires a single pass over all the patterns of the training set. This characteristic renders PNNs faster to train suitable for classification of power quality events. The architecture of PNN is composed of radial basis layer and competitive layer as shown in figure 5 .

\section{Hidden Layer}

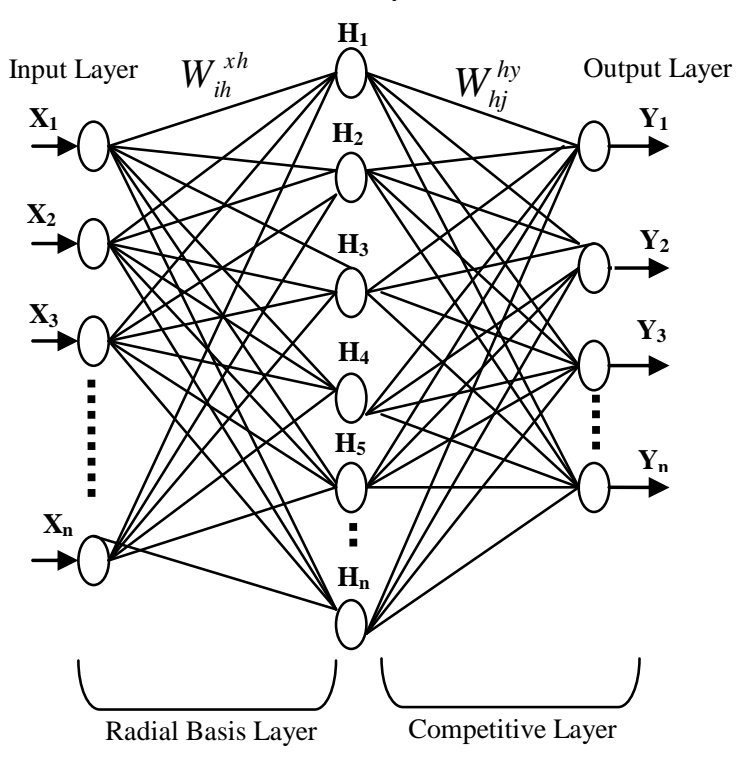

Figure 5. Architecture of PNN

For a classification application, the training data is classified according to their distribution values of probabilistic density function. A simple PDF is given by

$$
f_{k}(x)=\frac{1}{N_{k}} \sum_{j=1}^{N_{k}} \exp \left(-\frac{\left\|X-X_{k j}\right\|}{2 \sigma^{2}}\right)
$$

Modifying and applying (11) to the output vector $\mathrm{H}$ of the hidden layer in the PNN is given by

$$
H_{h}=\exp \left(\frac{-\sum_{i}\left(X_{i}-W_{i h}^{x h}\right)^{2}}{2 \sigma^{2}}\right)
$$

where

i number of input layers; 
$\mathrm{h}$ number of hidden layers;

j number of output layers;

$\mathrm{k}$ number of training examples;

$\mathrm{N}$ number of classifications (clusters);

$\sigma$ smoothing parameter (standard deviation);

$\mathrm{X}$ input vector;

$\| X-X_{k j} \mid$ Euclidean distance between the vectors $\mathrm{X}$ and $\mathrm{X}_{\mathrm{kj}}$;

$W_{i h}^{x h}$ connection weight between the input layer $\mathrm{X}$ and the hidden layer $\mathrm{H}$;

$W_{h j}^{h y}$ connection weight between the hidden layer $\mathrm{H}$ and the output layer $\mathrm{Y}$;

Figure 6. shows the block diagram for classification of voltage sag causes using PNN.

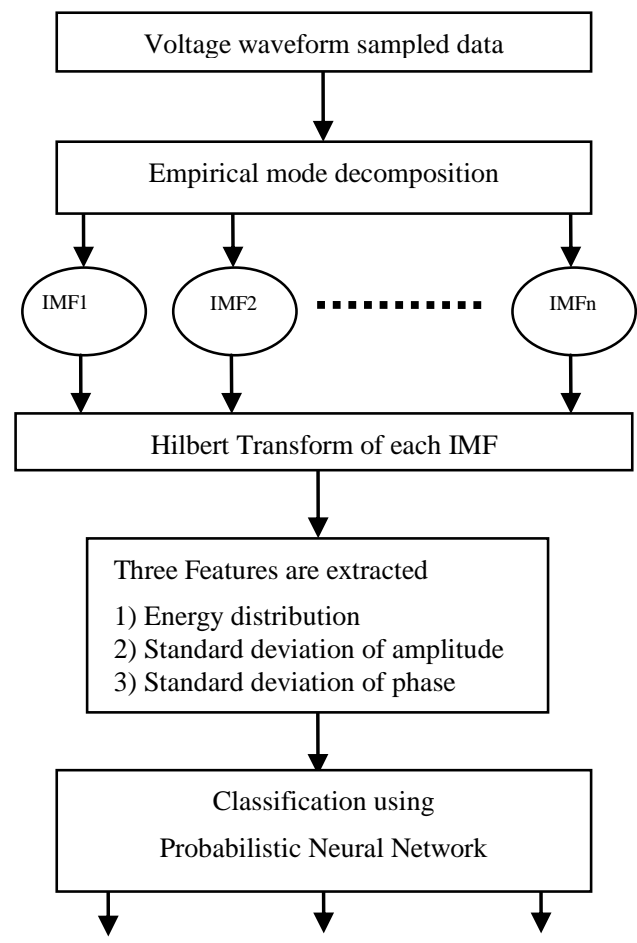

Figure 6. Block diagram for classification of voltage sag causes.

\section{RESULTS AND DISCUSSION}

Three voltage sag causes are taken they are (i) Three phase short circuit (ii) Starting of induction motor and (iii) Three phase transformer energization. The input signal is decomposed into number of modes by applying the Empirical mode decomposition. These decomposed mono component signals are called the IMFs. Hilbert transform of the first three IMFs is taken. Three features energy distribution, standard deviation of the amplitude and standard deviation of the phase of the three IMFs are extracted. These features are given as inputs to PNN. Simulations are performed to generate about 145 signals, 45 data set are used for training the PNN classifier and 100 are used for testing the classifier. When PNN is trained, the spread factor is tuned by trial and error method to 0.1, which has given better results. As shown in the Table.1 the PNN classifies all the testing samples accurately. However 3 disturbances events are classified incorrectly and the overall efficiency is $96.67 \%$.

Table 1. Classification results using PNN

\begin{tabular}{|l|c|c|c|}
\hline Cases & VSC1 & VSC2 & VSC3 \\
\hline VSC1 & 40 & 0 & 0 \\
\hline VSC2 & 0 & 27 & 3 \\
\hline VSC3 & 0 & 0 & 30 \\
\hline $\begin{array}{l}\text { Classification } \\
\text { efficiency in \% }\end{array}$ & 100 & 90 & 100 \\
\hline $\begin{array}{l}\text { Classification } \\
\text { error in \% }\end{array}$ & 0 & 10 & 0 \\
\hline Overall efficiency & & $96.67 \%$ & \\
\hline
\end{tabular}

\section{CONCLUSIONS}

In this paper PNN classifier combined with Hilbert transform is used to classify the type of voltage sag causes. EMD is used to decompose the original signal into mono component signals called the IMFs. Hilbert transform is applied to extract the features from first three IMFs. PNN classifier is used to classify the power quality disturbances. The results show that the method is efficient in classifying the voltage sag causes.

\section{REFERENCES}

[1] Damarla, G. P., Chandrasekaran, A., \& Sundaram, A. "Classification of power system disturbances through fuzzy neural network" in Electrical and Computer Engineering conference (pp. 68-71). Canada.owman, M., Debray, S. K., and Peterson, L. L. 1993.

[2] R. A. Flores, "State of art in the classification of power quality events, an overview,", in Proc. $10^{\text {th }}$ Int. Conf. Harmonics Quality Power,2002, vol. 1,pp.17-20.

[3] Y. H. Gu and M. H. J. Bollen, " Time-frequency and timescale domain analysis of voltage disturbances," IEEE Trans. Power Delivery, vol. 15, no.4 pp. 1279-1284, October 2000.

[4] F. Jurado, N. Acero, and B. Ogayar, "Application of signal processing tools for power quality ," in Proc. Canadian Conf. Electrical and Computer Engineering ,May 2002, vol.1,pp 82-87.

[5] S. Santoso, W. M. Grady, E. J. Powers, J. Lamoure, and S. C. Bhatt, "Characterization of distribution power quality events with Fourier and Wavelet transforms,"IEEE Trans. Power Delivery, vol.15, no.1,pp. 247-245,January 2000. 
[6] Z. L. Gaing , "Wavelet based neural network for power disturbance recognition and classification," IEEE Trans. Power Delivery, vol.19, no.4,pp.1560-1568,Oct. 2004.

[7] M.Gaouda, M.M.A.Salama and M.R.Sultan, A.Y.Chikhani, "Power Quality Detection and Classification Using Wavelet Multiressolution Signal Decomposition", IEEE Transactions on Power Delivery, Volume 14, Issue 4 October 1999, pp. 1469-1476.

[8] M. V. Chiukuri and P. K. Dash, "Multiresolution STransform based fuzzy recognition system for power quality events," IEEE Trans. Power Delivery ,vol.19,no.1, pp.323330,January 2004.

[9] N.E. Huang, Z. Shen, S.R. Long, M.C. Wu, H.H. Shih, Q. Zheng, N.C. Yen, C.C. Tung, H.H. Liu, The empirical mode decomposition and hilbert spectrum for nonlinear and nonstationary time series analysis, Proceedings of the Royal Society, London, Series A 454, 903-995, 1998.

[10] H. Amaris, C. Alvarez, M. Alonso, D. Florez, T. Lobos, P. Janik, J. Rezmer, Z. Waclawek," Application of advanced signal processing methods for accurate detection of voltage dips,' $13^{\text {th }}$ International Conference on Harmonics and Quality of Power, ICHQP 2008,Wollongong,Australia, pp.6 $28^{\text {th }}$ September 2008.

[11] S. Mishra, C. N. Bhende, and B. K. Panigrahi, " Detection and classification of power quality using S-transforms and probabilistic neural network, " IEEE Transactions on Power Delivery, Vol 23, Issue 1, January 2008, pp.280-287.284. 